\title{
Structural Changes in Liquid Crystals Doped with Rod-Like Magnetic Particles Studied by Surface Acoustic Waves
}

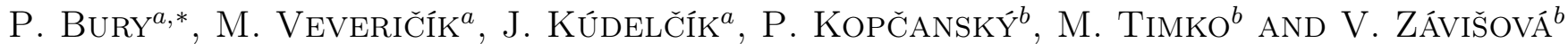 \\ ${ }^{a}$ Department of Physics, Žilina University, Univerzitná 1, 01026 Žilina, Slovakia \\ ${ }^{b}$ Institute of Experimental Physics, Slovak Academy of Sciences, Watsonova 47, 04001 Košice, Slovakia
}

The effect of rod-like magnetic particles on liquid crystal (6CHBT) structural changes in electric and weak magnetic fields were studied by means of the attenuation of surface acoustic wave of frequency $30 \mathrm{MHz}$ propagating along ferronematic liquid crystals. Three low volume concentrations $\left(\Phi=1 \times 10^{-5}, 1 \times 10^{-4}\right.$ and $\left.1 \times 10^{-3}\right)$ of rod-like magnetic particles were added to liquid crystal during its isotropic phase. Several measurements including the investigation of the effects of electrical and magnetic fields applied both separately and in conjunction as well as the influence of temperature on the surface acoustic wave attenuation were performed. The distinctive surface acoustic wave attenuation responses induced by both electric and magnetic fields in studied ferronematic liquid crystals have been observed suggesting both structural changes and the orientational coupling between magnetic moments of magnetic particles and the director of the liquid crystal. Obtained results confirmed the significant influence of the presence of magnetic particles on the structural properties and resulting behavior of 6CHBT liquid crystal.

DOI: 10.12693/APhysPolA.131.913

PACS/topics: $61.30 . \mathrm{Gd}, 75.50 . \mathrm{Mm}, 43.35 . \mathrm{Bf}$

\section{Introduction}

Liquid crystals (LCs) are anisotropic fluids with longrange orientational order, which combine the fluidity of ordinary liquids with the direction dependent electric and optical properties of crystalline solids. The major technical application of LCs is their use in the widespread liquid crystal displays. Nowadays scientists focus on different topics as for instance new possible use in optics, nanomicro manipulation, novel composites, nanosensors, and biotechnology. The doping process of LCs currently can prepare novel materials that can have very interesting properties which are absent in original LC substances.

There has been a continuously growing interest in this area of research and a number of interesting phenomena have been demonstrated. Composite systems, i.e. liquid crystals doped with different nanoparticles, such as gold, semiconducting, ferroelectric, magnetic particles or carbon nanotubes (CNTs) [1-4], are subjected to intensive research in an effort to produce materials with unique properties as well as to increase the sensitivity of these materials to external fields and study and explore the orientation of the particles in these systems.

Ferronematics are stable colloidal suspensions of fine magnetic particles in nematic liquid crystals. They attract considerable interest of investigators because their response to an external magnetic field considerably exceeds that of pure nematics. The most essential feature of these systems is a strong orientational coupling between the magnetic particles and the liquid crystal matrix $[5,6]$.

*corresponding author; e-mail: bury@fel.uniza.sk
Acoustic methods are useful tool for the characterization of LCs, particularly their elastic and viscous parameters in the vicinity of phase transitions. Concerning the surface acoustic waves (SAW) they were used to determine the viscosity distribution in LC layer depending on applied electric field, as the SAW-driven LC light shutter or SAW sensor [7,8]. In this contribution we present the utilization of SAW to study structural changes in nematic LCs doped with rod-like magnetic particles induced by electric and weak magnetic fields.

\section{Experimental}

Rod-like magnetic particles were synthesized through hydrolysis of $\mathrm{FeCl}_{3}$ and $\mathrm{FeSO}_{4}$ solutions (the $\mathrm{Fe}^{3+}$ to $\mathrm{Fe}^{2+}$ molar ratio was 2:1) containing urea. In a typical experiment $\mathrm{FeCl}_{3} \cdot 6 \mathrm{H}_{2} \mathrm{O}, \mathrm{FeSO}_{4} \cdot 7 \mathrm{H}_{2} \mathrm{O}$, and $\left(\mathrm{NH}_{2}\right)_{2} \mathrm{CO}$ were all dissolved in purified deoxygenated water. This mixture was added to a flask with reflux condenser and heated in a water bath for $12 \mathrm{~h}$ at $90-95^{\circ} \mathrm{C}$. A dark precipitate was formed. The sample was cleaned several times with purified and deoxygenated water, and then it was dried at a lower pressure at $50^{\circ} \mathrm{C}$ for $3 \mathrm{~h}$. The morphology and size distribution of the prepared nanorods were measured by transmission electron microscope Tesla BS 500. The sample dispersed in diluted ethanol was dropped on a copper grid and dried in air. The average diameter of rod-like particles was $80 \mathrm{~nm}$ and the mean length determined from the histogram of the size distribution was $1200 \mathrm{~nm}$ [9].

The surface acoustic waves of fundamental frequencies 10 and $20 \mathrm{MHz}$ were generated by an interdigital transducer (IDT) prepared on the $\mathrm{LiNbO}_{3}$ delay line using the Pulse Modulator and Receiver-MATEC 7700 and another IDT was used for receiving the surface wave. The acoustic attenuation was measured using Matec At- 
tenuation Recorder 2470 A. However, the harmonic frequency $30 \mathrm{MHz}$ appeared the most sensitive SAW frequency for structural changes study. The samples of LCs were placed on the top of the $\mathrm{LiNbO}_{3}$. Figure 1 shows the experimental arrangement, the LC layer $(D \approx 100 \mu \mathrm{m})$ was located on the center of the acoustic delay line and sandwiched between the delay line and glass plate, both coated with gold electrodes.

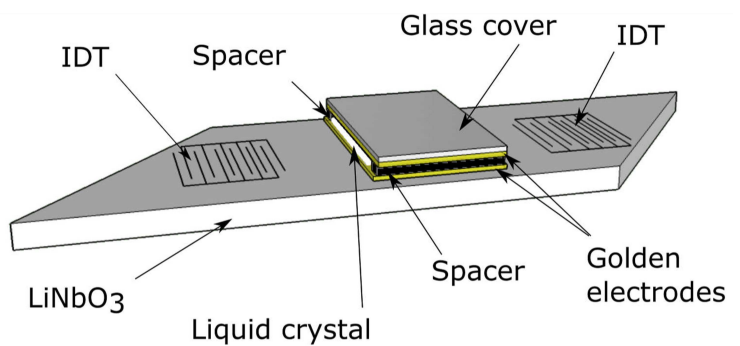

Fig. 1. Schematic arrangement of $\mathrm{LC}$ cell on $\mathrm{LiNbO}_{3}$ delay line for SAW investigation.

\section{Results and discussion}

As it was shown in our previous works [5, 9], there is an interaction between the magnetic moment of the magnetic particles and the liquid crystal molecules which favors a parallel initial orientation of magnetic moments and the director. The initial intrinsic arrangement of LC was supposed to have a planar alignment when the director $\boldsymbol{n}$ was parallel to the electrodes and electric field was then applied perpendicular to them. Structural changes in LC under both electric and magnetic fields were monitored using measurements of the attenuation of SAW propagating along the interface between the $\mathrm{LiNbO}_{3}$ delay line and LC cell. The applied electric field turned the director to its direction so that LC molecules changed orientation to perpendicular to the surface of electrodes and SAW attenuation subsequently changed. However, from the viscosity distribution measurement it follows that molecules change the orientation gradually and start at center [10].

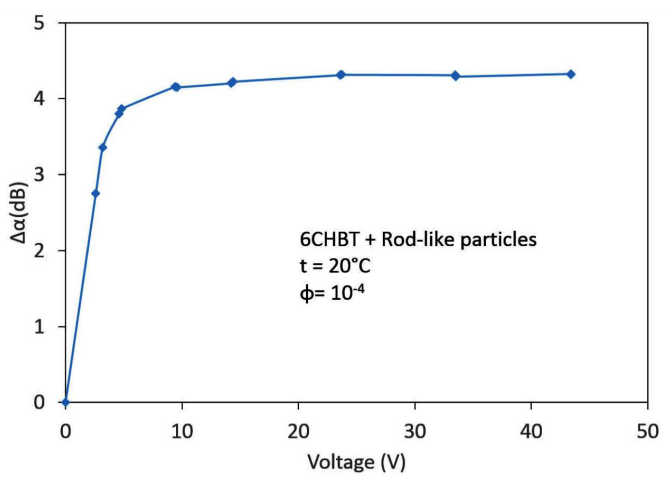

Fig. 2. Effect of applied voltage on SAW attenuation for 6 CHBT doped with rod-like particles $\left(\Phi=10^{-4}\right)$.

Figure 2 shows the effect of applied voltage on SAW attenuation of 6 CHBT doped with rod-like particles ( $\Phi=$ $\left.10^{-4}\right)$. It can be seen that the magnitude of this effect depends on the intensity of applied electric field and the reorientation of $\mathrm{LC}$ registered by acoustic attenuation is for voltages higher than $10 \mathrm{~V}$ practically saturated. Similar developments of acoustic attenuation depending on the applied electric field were detected also for different concentrations of magnetic particles. However, observed changes increased with the increase of particle concentration.

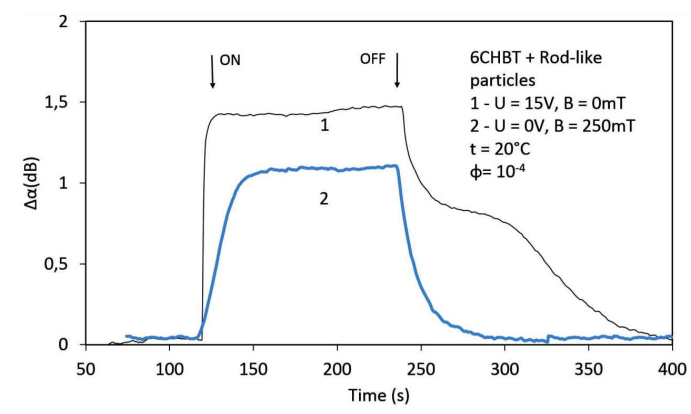

Fig. 3. Effects of applied voltage $(15 \mathrm{~V})$ and magnetic field $(250 \mathrm{mT})$ on SAW attenuation for doped $6 \mathrm{CHBT}$ $\left(\Phi=10^{-4}\right)$.

The progress of the switching processes and the comparison of effects of applied voltage $(15 \mathrm{~V})$ and magnetic field $(250 \mathrm{mT})$ on the SAW attenuation for doped $6 \mathrm{CHBT}$ $\left(\Phi=10^{-4}\right)$ is illustrated in Fig. 3. These developments indicate that LC molecules can be reoriented in the case of zero electric field also by magnetic field perpendicular to electrode surfaces, although with less effect. The larger time constants connected with structure changes in the case of applied magnetic field indicate also the presence of different process and the role of magnetic particles. The process of reorientation of magnetic particles is in this case influenced by both their coupling with LC molecules and probably some structure changes in particle forms [11]. While the relaxation time of the process occurring after the electric field activation was $1.04 \mathrm{~s}$, the relaxation time of process initiated by magnetic field was noticeably longer, $\tau=8.90 \mathrm{~s}$. While after the removing of magnetic fields only one process was registered with the relaxation time $119 \mathrm{~s}$, after removing of electric field two processes were evident. The first with relaxation time $9.10 \mathrm{~s}$, indicating very similar process as in the case of magnetic field and second with much longer relaxation time, $94.0 \mathrm{~s}$ that can be due to the larger magnetic particles. However, these processes are slower than in the case of spherical particles $[12,13]$. The experimental observations confirm the role of magnetic particles in LC and their interaction with liquid crystal molecules and coincide with previous results obtained investigating the dielectric behavior of the same LCs [5, 6, 9].

Three different successive applications of electric and/or magnetic fields are illustrated in Fig. 4. The lowest curve shows the application of magnetic $(250 \mathrm{mT})$ and electric $(15 \mathrm{~V})$ fields one after another. Another two curves show their application in opposite order but for 
voltages below saturation $(3 \mathrm{~V})$ and at saturation $(15 \mathrm{~V})$. While in the case of lower electric field the magnetic field contributes to the reorientation of LC molecules, but still not to the complete one, in the case of higher electric field the magnetic field has no effect. It means that though the magnetic field due to the coupling between magnetic moments of particles and LC molecules is able markedly contribute to the LC matrix reorientation, however not to saturated state.

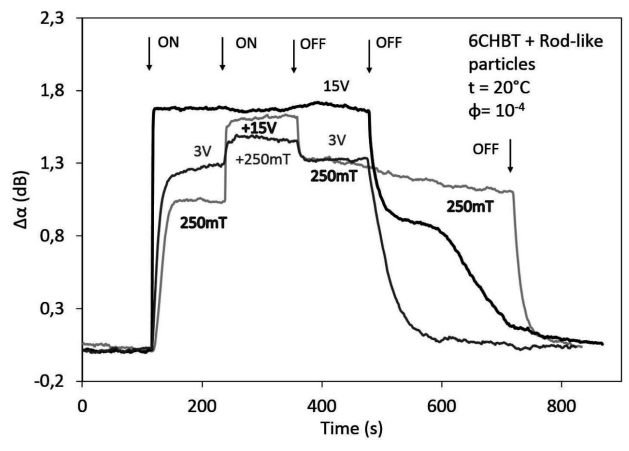

Fig. 4. Effect of gradual application of electric and/or magnetic fields on SAW attenuation for doped 6CHBT $\left(\Phi=10^{-4}\right)$.

The temperature dependence of acoustic attenuation measured for LC consisting of rod-like particles $(\Phi=$ $10^{-4}$ ) (Fig. 5) shows after the initial increase of acoustic attenuation up to temperature corresponding to the melting point the decrease that could indicate the role of thermal motion on the interaction of surface acoustic wave and LC molecules. This fact is supported also by changes of the SAW attenuation during $3 \mathrm{~V}$ pulses. No well-marked structural transition was registered at the clearing point.

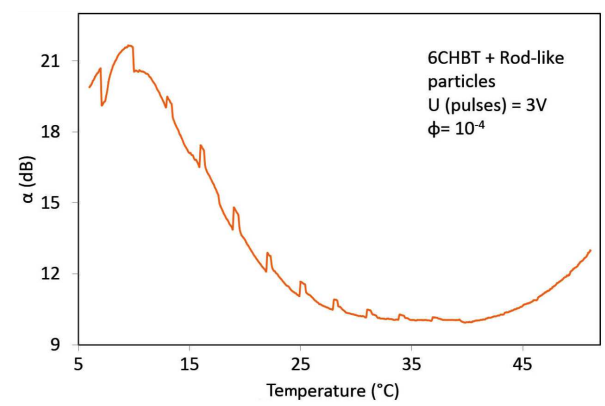

Fig. 5. Temperature dependence of SAW attenuation for doped 6CHBT $\left(\Phi=10^{-4}\right)$ with alternating application of voltage pulses $3 \mathrm{~V}$.

\section{Conclusions}

In this contribution we have presented the utilization of SAW to study the structure changes in 6CHBT doped with rod-like magnetic particles, induced by electric and weak magnetic fields. Obtained results acknowledged previously supposed orientational coupling between magnetic particles and liquid crystal matrix. Doping of a nematic liquid crystal with rod-like magnetic particles causes an effective orientational coupling between the magnetic moments of magnetic particles and the director of the nematic liquid crystal. The obtained results showed that in ferronematics based on the 6CHBT liquid crystal, the orientation of the magnetic moments of magnetic particles and molecules of the liquid crystal are parallel. However, the director of the liquid crystal at some distance from the surface of the magnetic particles in the droplets depends on the strength of the applied external fields. In conclusion, our results showed that the combination of electric and magnetic fields enables the better control of the orientation of LC molecules in ferronematic samples than only a single field.

\section{Acknowledgments}

This work was supported by VEGA project 2/0045/13 and the R\&D operational program Centrum of excellence of power electronics systems and materials for their components, No. OPVaV-2008/2.1/01-SORO, ITMS 26220120003 and 26220120021 funded by European Community. Authors also would like to thank to Mr. František Cernobila for technical assistance.

\section{References}

[1] F. Li, O. Buchnev, C.I. Cheon, A. Glushchenko, V. Reshetnyak, Y. Reznikov, T.J. Sluckin, J.L. West, Phys. Rev. Lett. 97, 147801 (2006).

[2] T.Z. Shen, S.H. Hong, J.K. Song, Nat. Mater. 13 , 394 (2014).

[3] B. Kinkead, T. Hengman, J. Mater. Chem. 20, 448 (2010).

[4] M. Rahman, W. Lee, J. Phys. D Appl. Phys. 42, 063001 (2009).

[5] N. Tomašovičová, P. Kopčanský, M. Koneracká, V. Závišová, M. Timko, N. Éber, T. Tóth-Katona, J. Jadzyn, J. Phys. Condens. Matter 20, 204123 (2008).

[6] N. Tomašovičová, M. Timko, V. Závišová, A. Hashim, J. Jadzyn, X. Chaud, E. Beaugnon, P. Kopčanský, Int. J. Thermophys. 35, 2044 (2014).

[7] Y.J. Liu, X. Ding, Sz-Chin Steven Lin, J. Shi, I-Kao Chiang, T.J. Huang, Adv. Mater. 23, 1656 (2011).

[8] S.J. Patrash, E.T. Zellers, Anal. Chim. Acta 288, 167 (1994).

[9] P. Kopčanský, N. Tomašovičová, M. Koneracká, V. Závišová, M. Timko, A. Džáavá, P. Sprincová, Phys. Rev. E 78, 011702 (2008).

[10] H. Moritake, R. Ozaki, X. Chiba, H. Yamamoto, J. Ogawa, K. Yoshino, in: IEEE Int. Conf. on Dielectric Liquids, Trondheim 2011.

[11] J. Kúdelčík, P. Bury, P. Kopčanský, M. Timko, J. Magn. Magn. Mater. 388, 28 (2015).

[12] P. Bury, Š. Hardoň, J. Kúdelčík, M. Timko, P. Kopčanský, Phys. Proced. 75, 1022 (2015).

[13] P. Bury, Š. Hardoň, J. Kúdelčík, M. Veveričík, P. Kopčanský, M. Timko, V. Závišová, J. Magn. Magn. Mater. 423, 57 (2017). 